\title{
Validation of the Backscattered Frequency Correlation Function for the Inversion of Biophysical Parameters from Natural Targets
}

\author{
J. M. López-Sánchez ${ }^{1} \quad$ J. Fortuny-Guasch ${ }^{1} \quad$ A. J. Sieber ${ }^{1}$ K. Sarabandi ${ }^{2}$ \\ 1 Advanced Techniques, SAI JRC, TP.720, 21020 Ispra (Va), Italy \\ Tel/Fax: +39332 785104/5772, E-mail: Juan.Lopez-Sanchez@jrc.it \\ ${ }^{2}$ Department of Electrical Engineering and Computer Science \\ The University of Michigan, Ann Arbor, MI 48109-2122
}

\begin{abstract}
The computation of the frequency correlation function (FCF) in wide-band measurements of the radar backscatter from complex distributed targets allows the estimation of parameters related to the structure and geometry of the targets, since the individual response of the single components has slight variations with frequency. In this paper, the application of this technique to data acquired in a laboratory under fully controlled conditions is described. The FCF of the distributed target has clear trends and features, as the main lobe width and the position and level of the secondary lobes, which can be used to characterize and classify it. The targets used in this experimental validation include two samples of maize crops, a fir tree and a ficus plant. The height and density of the maize plants are parameters expected to be inverted, but an analytical or numerical method for this inversion has not been developed yet. The FCF could be also employed to identify different vegetation kinds, since it is sensitive to the plant structure.
\end{abstract}

\section{INTRODUCTION}

In recent years, some approaches for increasing the number of estimable parameters from remote sensing measurements have been developed. These techniques employ multi-frequency, multi-polarization or interferometric systems. An effective approach consists on the computation of the FCF [1] of the radar backscatter response in order to retrieve physical parameters from natural targets. The usefulness of this technique for distributed targets is based on the fact that the scattering from a given element changes only slightly with frequency, while the entire target decorrelates faster. This is due to that frequency decorrelation depends on changes in the relative positions between scatterers (phase changes), and not on the scatterers themselves.

In [1] the backscattered FCF was applied to estimate the rms height of rough surfaces, and the extinction rate and height of grass fields and snow layers. This type of targets has in common a definite interface between free space and the medium which constitutes the target. For such targets it is relatively easy to compute the system
FCF, which only depends on the measurement instrumentation and geometry [2]. Later, this system FCF can be removed from the measured FCF and the target FCF is obtained. Therefore, the target FCF only depends on the target itself and the estimation of its physical parameters becomes straightforward. In this paper we have started to investigate the application of the backscattered FCF to vegetation samples. Vegetation targets have a very complex geometry and we have not found yet the way for computing a system FCF matched to these targets. Anyway, it is possible to compare coherently the measured FCF from different targets if the same measurement setup is employed for all targets. Then, we can obtain useful information for estimating physical parameters or identifying different vegetation kinds by knowing that there is always a common dependence on the system.

In the first section, the theoretical definition of the backscattered FCF and the way to compute it in real measurements are briefly presented. The second section shows the experimental setup, the results we have obtained and the discussion about the potential of this technique.

\section{BACKSCATTERED FREQUENCY CORRELATION FUNCTION}

Consider a radar system operating in stepped frequency mode which transmits in $N_{f}$ frequency steps over a total bandwidth $B$. If the bandwidth is a small fraction of the center frequency $f_{0}$, the backscattering coefficient will be approximately independent of frequency:

$$
\left\langle|E(f)|^{2}\right\rangle \simeq\left\langle\left|E\left(f_{0}\right)\right|^{2}\right\rangle
$$

Thus the backscatter process $E(f)$ becomes a stationary process and its covariance only depends on the frequency shift:

$$
C\left(f_{1}, f_{2}\right)=\left\langle E\left(f_{2}\right) E^{*}\left(f_{1}\right)\right\rangle=C\left(f_{2}-f_{1}\right)
$$

The FCF is defined as the normalized covariance function. Its magnitude and phase are:

$$
R(\Delta f)=\left|\frac{C(\Delta f)}{C(0)}\right| \quad \Phi(\Delta f)=\angle \frac{C(\Delta f)}{C(0)}
$$


When $\Delta f=0$ the FCF is maximum, and approaches zero as the $\Delta f$ increases. In practice, the statistical averaging is obtained by measuring $N_{s}$ independent samples, and the FCF is computed in the following way:

$$
C(\Delta f)=\frac{1}{N_{s}} \sum_{i=1}^{N_{s}} \frac{1}{N_{f}-M} \sum_{j=1}^{N_{f}-M}\left(E_{i, j} E_{i, j+M}^{*}\right)
$$

where $E_{i, j}$ denotes the electric field of the $i$ th sample at the $j$ th frequency point, and $\Delta f=M B / N_{f}$.

\section{EXPERIMENTAL RESULTS}

The experiment consisted of microwave radar measurements on different vegetation samples. These measurements were performed in a controlled environment by making use of the anechoic chamber of the European Microwave Signature Laboratory (EMSL) at JRC Ispra in Italy. The samples were mounted on a rotating platform inside the anechoic chamber. The container was covered with microwave absorber, to make sure that the measured backscatter did not include any contribution from the ground and the corresponding interaction with the plants. The measurement system used in this experiment is based on a network analyzer and operates in the stepped-frequency mode. All the measurements were carried out within a wide frequency range and an incidence elevation angle span. The acquired data in the frequency domain were empty room subtracted and gated in the time domain in order to isolate the response of the targets from the residual antennas coupling and eventual spurious reflections in the chamber. The targets were rotated on the turntable in order to obtain the statistical averaging needed for the FCF definition. Here we present results from spans of $1 \mathrm{GHz}$ centered at $\mathrm{S}, \mathrm{C}$ and $\mathrm{X}$ bands: $2.4-$ $3.4,4.5-5.5$ and $7.8-8.8 \mathrm{GHz}$ respectively.

\section{Groups of plants: maize samples}

The first result is the comparison of the FCF obtained from two samples of maize crops. The targets are two groups of $6 \times 6$ plants about $1.2 \mathrm{~m}$ high and $4 \times 4$ plants about $1.6 \mathrm{~m}$ high positioned on a square container. Results were computed at $\mathrm{S}$ band for incidence angles from $0^{\circ}$ to $60^{\circ}$. Fig. 1 shows the results for $0^{\circ}, 30^{\circ}$ and $60^{\circ}$. Note that the measurement system and geometry are the same in both cases, thus a consistent comparison is possible without taken into account the influence of the system. At 0 degrees (from the top of the target) the FCF for both copolar responses are the same, due to that from the top the radar is sensible only to the highest leaves of the maize plants, which are randomly distributed and oriented. It is also clear that the FCF of the first sample has higher oscillations. It may be produced by the lower density of the second sample, since it is the only different parameter in both cases (the length of the trunks has no influence on a top view). For non-zero incidence angles, all the results show a higher level on the second lobe for the $H H$ than for the $V V$. It is the expected behavior due to the influence of the ground, which reduces the vertical polarization response. As the incidence angle increases, the $H H$ curves do not change very much, but the $V V$ curves present varying trends. It may be caused by the presence of the ground and by the vertical trunks of the plants, which are visible from these angles. Finally, it is evident that the FCF from both samples are quite different for high elevation angles because the plants have different heights.

\section{Single plants: fir tree and ficus}

From the previous results it can be stated that the FCF technique is very sensitive to the physical structure of the sample. We have further investigated this fact by comparing the measurements at $\mathrm{C}$ band from two single trees: a fir tree and a ficus plant. The fir tree used in this experiment was about $5 \mathrm{~m}$ high and $2.5 \mathrm{~m}$ wide. This tree presented quasi-horizontal branches bearing typically $2-3 \mathrm{~cm}$ needles showing a brush-like distribution. The branches constituted large horizontal planar surfaces at different levels in height. The top of the tree conformed to a young tree of its same species. The ficus tree was about $2.5 \mathrm{~m}$ high and $1 \mathrm{~m}$ wide. This tree showed 10 to $25 \mathrm{~cm}$ long pointed leaves and many-twigged slender branches. Moreover, the effect of the ground plane has been observed by repeating the measurement from the ficus by positioning the pot on an absorber flat plane instead of being isolated and covered by pieces of pyramidal absorbers. Both results from the ficus are presented in Fig.2, showing a completely different response due to the ground plane presence. The level of the first secondary lobe is higher for $H H$ than $V V$ when the ground plane is present. Results computed from the fir tree are presented in Fig. 3 for 30 to 50 degrees. Comparing the result for 50 degrees with the one from the ficus, it is clear that the FCF is sensitive to the change of tree morphology as expected.

\section{CONCLUSIONS}

Some preliminary results have been presented, showing the potentials of the FCF for identifying different plants kinds and different biophysical parameters of the same kind. A construction of inversion methods is still needed, but the first insights are promising.

\section{REFERENCES}

[1] K. Sarabandi and A. Nashashibi, "Analysis and Applications of Backscattered Frequency Correlation Function," IEEE Trans. Antennas Propagat., in press.

[2] K. Sarabandi, Y. Oh, and F. T. Ulaby, "Measurement and Calibration of Differential Mueller Matrix of Distributed Targets," IEEE Trans. Antennas Propagat., vol. 40, pp. 1524-1532, Dec 1992. 

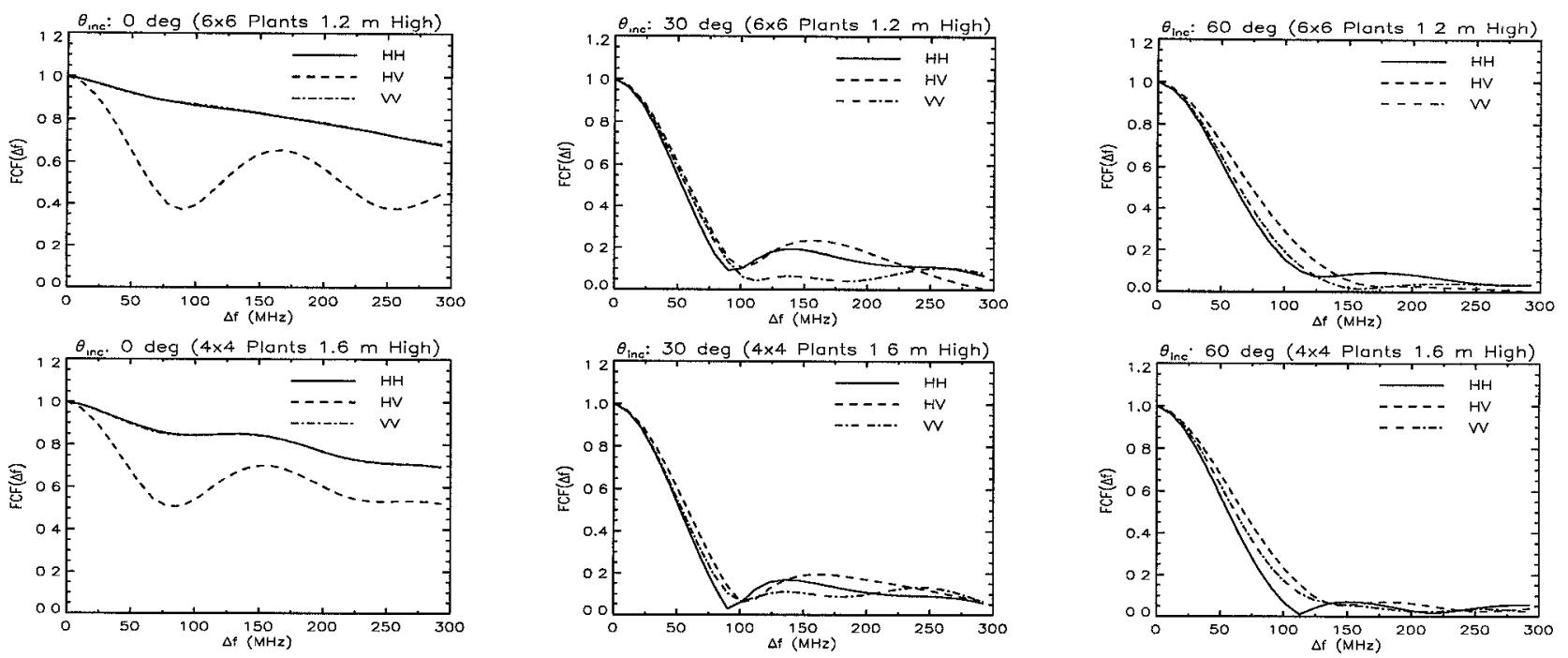

Figure 1: Backscattered FCF from the maize samples at $\mathrm{S}$ band. $\theta_{i}=0^{\circ}, 30^{\circ}, 60^{\circ}$
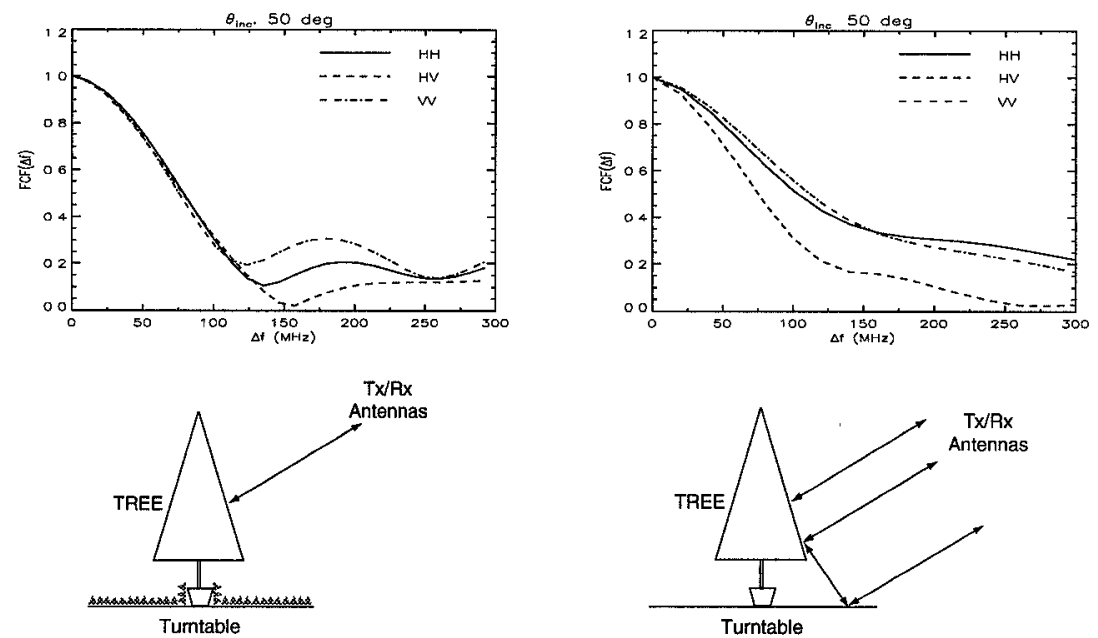

Figure 2: Backscattered FCF from the ficus tree at $\mathrm{C}$ band. $\theta_{2}=50^{\circ}$. Cases: isolated pot (left) and pot on a ground plane (right)
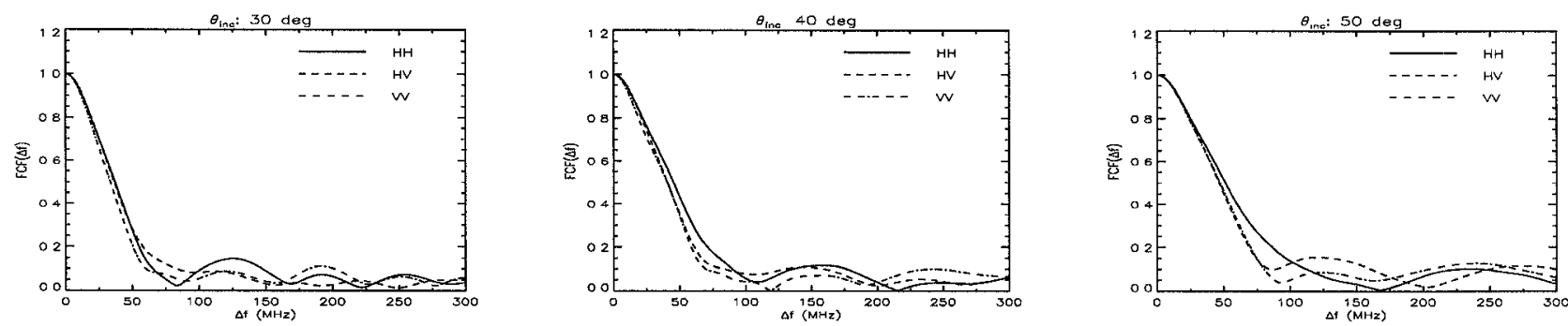

Figure 3: Backscattered FCF from the fir tree at C band. $\theta_{i}=30^{\circ}-50^{\circ}$ 